\title{
A Numerical Approach for Computing Standard Errors of Linear Equating
}

\author{
Lingjia Zeng \\ American College Testing
}

\begin{abstract}
A numerical approach for computing standard errors (SES) of a linear equating is described. In the proposed approach, the first partial derivatives of the equating function needed to compute the SEs are derived numerically. Thus, the difficulty of deriving the analytical formulas of the partial derivatives for a complicated equating method is avoided. The numerical and analytical approaches were compared using the Tucker equating method. The SEs derived numerically were found to be
\end{abstract}

\begin{abstract}
indistinguishable from the SEs derived analytically. In a computer simulation of the numerical approach using the Levine equating method, the SEs based on the normality assumption were found to be less accurate than those derived without the normality assumption when the score distributions were skewed. Index terms: common-item design, Levine equating method, linear equating, standard error of equating, Tucker equating method.
\end{abstract}

A number of authors have derived standard errors (SEs) of linear equatings. Lord (1950) reported formulas for computing the SES of linear equatings under a variety of different designs. Lord's SE formulas were derived with a strong assumption that the scores of the tests being equated have a normal bivariate distribution in the population from which the sample is drawn. Braun \& Holland (1982) and Kolen (1985) suggested that SEs based on the normality assumption might produce misleading results when the score distributions are skewed or more peaked than a normal distribution. SEs of linear equating derived from a general delta method without making a normality assumption were reported by Braun \& Holland (1982) for the randomly equivalent groups design, and by Kolen (1985) for the Tucker equating method (Angoff, 1971, pp. 579-583).

The delta method, as described by Kendall \& Stuart (1977, pp. 246-247), is a general method for deriving the sampling variance of a function of random variables. The delta method is derived from a first-order Taylor's series. By using the delta method, the sampling variance of a linear equating can be derived from the first partial derivatives of the linear equating function and the variances and covariances of the moments that are involved in the equating function. The analytical expressions for the first partial derivatives of the equating function can be fairly simple for a simple equating function such as linear equating with the random groups design (see Braun \& Holland, 1982), or very complicated for a complicated equating function such as one using the common-itemnonequivalent-groups (CING) design (see Kolen, 1985). Using numerically derived derivatives is an easy way to circumvent the difficulty of deriving the analytical formulas needed to compute SEs of an equating. Lord (1975) presented an example of using numerical derivatives in computing asymptotic sampling variances.

A numerical approach for computing SEs of linear equating is described. In the proposed approach, the first partial derivatives of the equating function are computed numerically. The assumption of normality is optional when using the proposed approach for computing SEs of a linear equating. 
The accuracy of the numerical derivatives was examined using the Tucker equating method. The proposed approach also was applied to the Levine equating method.

\section{Method}

Consider a linear equating function $l(x)$ that transforms score $x$ of one test form, say Form $\mathrm{X}$, to the scale of another form, say Form $\mathrm{Y}$. In linear equating, the function $l(x)$ is based on $p$ random variables that are the central moments of the score distributions of Forms $\mathrm{X}$ and $\mathrm{Y}$. For example, in a random groups design, $l(x)$ involves four moments-the means and variances of the score distributions of the two test forms. In the CING design, $l(x)$ is based on 10 moments of the bivariate score distributions of the two forms. Let $\theta_{1}, \theta_{2}, \ldots, \theta_{p}$ be the $p$ central moments involved in $l(x)$, and $\hat{\theta}_{1}, \hat{\theta}_{2}, \ldots, \hat{\theta}_{p}$ be their estimates. According to Kendall \& Stuart (1977), the sampling variance of $\hat{l}(x)$, which is an estimate of the linear equating function $l(x)$, can be estimated by:

$\operatorname{var}\left[\hat{l}\left(x \mid \theta_{1}, \ldots, \theta_{p}\right)\right]=\sum_{i=1}^{p}\left(\frac{\partial l}{\partial \theta_{l}}\right)^{2} \operatorname{var}\left(\hat{\theta}_{l}\right)+\sum_{i=1}^{p} \sum_{j \neq l=1}^{p} \frac{\partial l}{\partial \theta_{l}} \frac{\partial l}{\partial \theta_{j}} \operatorname{cov}\left(\hat{\theta}_{l}, \hat{\theta}_{j}\right)+r$,

where

$\partial l / \partial \theta_{i}$ is the partial derivative of the linear equating function $l$, with respect to moment $\theta_{i}$, $\operatorname{var}\left(\hat{\theta}_{i}\right)$ is the variance of $\hat{\theta}_{i}$, $\operatorname{cov}\left(\hat{\theta}_{t}, \hat{\theta}_{j}\right)$ is the covariance of $\hat{\theta}_{i}$ and $\hat{\theta}_{j}$, and

$r$ is the higher-order remainder term, which is small and can be ignored.

The SE of $\hat{l}(x)$ is the square root of $\operatorname{var}\left[\hat{l}\left(x \mid \theta_{1}, \theta_{2}, \ldots, \theta_{p}\right)\right]$. Moment estimates $\hat{\theta}_{1}, \hat{\theta}_{2}, \ldots, \hat{\theta}_{p}$ may be obtained from one sample as in a single-group design, two samples as in a random-groups design or CING design, or three or more samples as in a chain of equatings. If $\hat{\theta}_{1}$ and $\hat{\theta}_{j}$ are moment estimates obtained from two independent samples, then $\operatorname{cov}\left(\hat{\theta}_{t}, \hat{\theta}_{j}\right)$ is 0 . When two or more samples are used, Equation 1 can be rewritten to exclude all the 0 terms from the summation.

The computation of the variances and covariances of the moments needed in Equation 1 depends on what data collection design is used in equating. In the random-groups design, the moments used in the equating functions are obtained from univariate distributions. The computation of variances and covariances of univariate moments is straightforward. However, for the CING design or singlegroup design, the variances and covariances of bivariate moments need to be found. Kendall \& Stuart $(1977$, p. 250$)$ described a general method for deriving the variance and covariance of bivariate moments. Using Kendall and Stuart's method, Kolen (1985) derived formulas for computing the variances and covariances of bivariate moments for equatings with the CING design (see Table 1). The formulas in the column labeled "normal" assume that the score distributions are bivariate normal. The formulas in the column labeled "general" do not make such an assumption. These formulas were incorporated with Equation 1 to compute the SEs for the Tucker and Levine equating methods in this study.

The Lord (1950), Braun \& Holland (1982), and Kolen (1985) derivations solved the first partial derivatives analytically. However, the first partial derivatives needed in Equation 1 also can be computed numerically. There are a number of formulas available for numerical differentiation, and they can be found in many numerical analysis textbooks (e.g., Burden \& Faires, 1985, pp. 140-141). Let $\theta$ denote a vector of moments $\theta_{r}, i=1$ to $p$, where $p$ is the total number of moments used in $l(x)$. Then, the first partial derivative of $l(x)$ with respect to $\theta_{i}$ can be approximated by 


\section{Table 1}

Sampling Variances and Covariances of Bivariate Moments Based on Large Sample Theory (From Kolen, 1985)

\begin{tabular}{lll}
\hline \hline & \multicolumn{2}{c}{ Sampling Variance and Covariance } \\
\cline { 2 - 3 } Statistic & General & Normal \\
\hline $\operatorname{var}[\hat{\mu}(\mathrm{X})]$ & $\sigma^{2}(\mathrm{X}) / n$ & $\sigma^{2}(\mathrm{X}) / n$ \\
$\operatorname{var}\left[\hat{\sigma}^{2}(\mathrm{X})\right]$ & $\left\{\mathrm{E}[\mathrm{X}-\mu(\mathrm{X})]^{4}-\sigma^{4}(\mathrm{X})\right\} / n$ & $2 \sigma^{4}(\mathrm{X}) / n$ \\
$\operatorname{var}[\hat{\sigma}(\mathrm{X}, \mathrm{V})]$ & $\left\{\mathrm{E}[\mathrm{X}-\mu(\mathrm{X})]^{2}[\mathrm{~V}-\mu(\mathrm{V})]^{2}-\sigma^{2}(\mathrm{X}, \mathrm{V})\right\} / n$ & {$\left[\sigma^{2}(\mathrm{X}) \sigma^{2}(\mathrm{~V})+\sigma^{2}(\mathrm{X}, \mathrm{V})\right] / n$} \\
$\operatorname{cov}[\hat{\mu}(\mathrm{X}), \hat{\mu}(\mathrm{V})]$ & $\sigma(\mathrm{X}, \mathrm{V}) / n$ & $\sigma(\mathrm{X}, \mathrm{V}) / n$ \\
$\operatorname{cov}\left[\hat{\mu}(\mathrm{X}), \hat{\sigma}^{2}(\mathrm{X})\right]$ & $\mathrm{E}[\mathrm{X}-\mu(\mathrm{X})]^{3} / n$ & 0 \\
$\operatorname{cov}\left[\hat{\mu}(\mathrm{X}), \hat{\sigma}^{2}(\mathrm{~V})\right]$ & $\mathrm{E}[\mathrm{X}-\mu(\mathrm{X})][\mathrm{V}-\mu(\mathrm{V})]^{2} / n$ & 0 \\
$\operatorname{cov}[\hat{\mu}(\mathrm{X}), \hat{\sigma}(\mathrm{X}, \mathrm{V})]$ & $\left\{\mathrm{E}[\mathrm{X}-\mu(\mathrm{X})]^{2}[\mathrm{~V}-\mu(\mathrm{V})]\right\} / n$ & 0 \\
$\operatorname{cov}\left[\hat{\sigma}^{2}(\mathrm{X}), \hat{\sigma}^{2}(\mathrm{~V})\right]$ & $\left\{\mathrm{E}[\mathrm{X}-\mu(\mathrm{X})]^{2}[\mathrm{~V}-\mu(\mathrm{V})]^{2}-\sigma^{2}(\mathrm{X}) \sigma^{2}(\mathrm{~V})\right\} / n$ & $2 \sigma^{2}(\mathrm{X}, \mathrm{V}) / n$ \\
$\operatorname{cov}\left[\hat{\sigma}^{2}(\mathrm{X}), \hat{\boldsymbol{\sigma}}(\mathrm{X}, \mathrm{V})\right]$ & $\left\{\mathrm{E}[\mathrm{X}-\mu(\mathrm{X})]^{3}[\mathrm{~V}-\mu(\mathrm{V})]-\sigma^{2}(\mathrm{X}) \sigma(\mathrm{X}, \mathrm{V})\right\} / n$ & $2 \sigma(\mathrm{X}, \mathrm{V}) \sigma^{2}(\mathrm{X}) / n$ \\
\hline
\end{tabular}

$\frac{\partial l}{\partial \theta_{i}}(x \mid \boldsymbol{\theta})=\frac{l\left(x \mid \theta+\delta_{i}\right)-l\left(x \mid \theta-\delta_{i}\right)}{2 h_{i}}-O\left(h_{i}^{2}\right)$,

where $O\left(h_{i}^{2}\right)$ is the error of the approximation, $\delta_{i}$ is the $i$ th row of the diagonal matrix $\Delta$, and

$\boldsymbol{\Delta}=\left[\begin{array}{lllll}h_{1} & & & & \\ & h_{2} & & \\ & & \ldots & \\ & & & \ldots & \\ & & & & h_{p}\end{array}\right]$,

where $h_{i}$ is a small value. Here, $h_{i}$ is set to $\theta_{i} / 1,000$. Because Equation 2 is derived by expanding $l(x \mid \theta)$ at two neighboring points $\left(\boldsymbol{\theta}+\boldsymbol{\delta}_{i}\right)$ and $\left(\boldsymbol{\theta}-\boldsymbol{\delta}_{i}\right)$ with a second-order Taylor's series, the magnitude of the error is based on the magnitude of the third partial derivative. If the third partial derivative with respect to $\theta_{i}$ is 0 , then the first partial derivative with respect to $\theta_{i}$ approximated by Equation 2 is the exact value; otherwise, error is involved in the approximation. The error of approximation is bounded by $C h_{i}^{2}$ where $C$ is the maximum absolute value of the third partial derivative with respect to $\theta_{i}$.

Equation 2 is known as the three-point approximation formula. A more accurate method is the five-point approximation formula, which involves evaluating $l(x)$ at more points and has the following form:

$\frac{\partial l}{\partial \theta_{i}}(x \mid \theta)=\frac{1}{12 h_{i}}\left[l\left(x \mid \theta-2 \delta_{i}\right)-8 l\left(x \mid \theta-\delta_{i}\right)+8 l\left(x \mid \theta+\delta_{i}\right)-l\left(x \mid \theta+2 \delta_{i}\right)\right]+O\left(h_{i}^{4}\right)$.

Equation 4 is derived by expanding $l(x \mid \boldsymbol{\theta})$ at four neighboring points- $\left(\boldsymbol{\theta}-2 \boldsymbol{\delta}_{i}\right),\left(\boldsymbol{\theta}-\boldsymbol{\delta}_{i}\right),\left(\boldsymbol{\theta}+\boldsymbol{\delta}_{i}\right)$, and $\left(\theta+2 \delta_{i}\right)$-with a fourth-order Taylor's series. The error term in Equation 4 is $O\left(h_{i}^{4}\right)$, which means that the error of approximation is bounded by $C h_{i}^{4}$, where $C$ is the maximum absolute value of the fifth partial derivative with respect to $\theta_{i}$.

In summary, given a linear equating function $l$, the sampling variance of the linear equating 
function $\hat{l}(x)$ can be computed with the following steps:

1. Find the estimates of the variances and covariances of the $p$ moments that are used in $\hat{l}(x)$.

2. Find the first partial derivatives with respect to all the $p$ moments evaluated at $x$.

3. Compute $\operatorname{var}[\hat{l}(x)]$ by substituting estimates in the first partial derivatives and in the variances and covariances into Equation 1.

These three steps can be incorporated easily into a computer program to perform equating and to compute SES.

\section{Accuracy of the Numerical Approach}

The accuracy of the SES of equating computed with the numerical approach was examined using the Tucker equating function described by Kolen \& Brennan (1987; see Table 2). In Table 2, $w_{1}$ and $w_{2}$ are used to weight the strata in defining the synthetic population. Conceptually, the synthetic population contains two strata-Population 1 and Population 2. Examinees administered the new form are considered to be a random sample from Population 1 , and examinees administered the old form are considered to be a random sample from Population 2. The two populations are proportionally weighted by $w_{1}$ and $w_{2}$, where $w_{1}+w_{2}=1$ and $w_{1}, w_{2} \geq 0$. Here, $w_{1}$ and $w_{2}$ were set equal (i.e., $w_{1}=w_{2}=.5$ ). More detailed discussion about the synthetic population and weights can be found in Kolen \& Brennan (1987). Kolen (1985) described an analytical approach for computing SES of Tucker equating. The analytical derivatives and the SEs of Tucker equating based on the analytical derivatives were used in this example as criteria to evaluate the accuracy of the numerical approach.

\section{Table 2}

General Equations for Common-Item Linear Equating with

Nonequivalent Populations (From Kolen \& Brennan, 1987)

$$
\begin{aligned}
& l(x)=\frac{\sigma_{s}(\mathbf{Y})}{\sigma_{s}(\mathbf{X})}\left[x-\mu_{s}(\mathbf{X})\right]+\mu_{s}(\mathrm{Y}), \text { where } \\
& \mu_{s}(\mathbf{X})=\mu_{1}(\mathbf{X})-w_{2} \gamma_{1}\left[\mu_{1}(\mathrm{~V})-\mu_{2}(\mathrm{~V})\right] \\
& \mu_{s}(\mathbf{Y})=\mu_{2}(\mathbf{Y})-w_{1} \gamma_{2}\left[\mu_{1}(\mathrm{~V})-\mu_{2}(\mathrm{~V})\right] \\
& \sigma_{S}^{2}(\mathbf{X})=\sigma_{1}^{2}(\mathbf{X})-w_{2} \gamma_{1}^{2}\left[\sigma_{1}^{2}(\mathrm{~V})-\sigma_{2}^{2}(\mathrm{~V})\right]+w_{1} w_{2} \gamma_{1}^{2}\left[\mu_{1}(\mathrm{~V})-\mu_{2}(\mathrm{~V})\right]^{2} \\
& \sigma_{s}^{2}(\mathbf{Y})=\sigma_{2}^{2}(\mathbf{Y})+w_{1} \gamma_{2}^{2}\left[\sigma_{1}^{2}(\mathrm{~V})-\sigma_{2}^{2}(\mathrm{~V})\right]+w_{1} w_{2} \gamma_{2}^{2}\left[\mu_{1}(\mathrm{~V})-\mu_{2}(\mathrm{~V})\right]^{2}
\end{aligned}
$$

Tucker Method

$$
\begin{aligned}
& \gamma_{1}=\frac{\sigma_{1}(\mathrm{X}, \mathrm{V})}{\sigma_{1}^{2}(\mathrm{~V})} \\
& \gamma_{2}=\frac{\sigma_{2}(\mathrm{Y}, \mathrm{V})}{\sigma_{2}^{2}(\mathrm{~V})}
\end{aligned}
$$

Levine Method for Exclusive Equating Section

$$
\begin{aligned}
& \gamma_{1}=\frac{\sigma_{1}^{2}(\mathbf{X})+\sigma_{1}(\mathbf{X}, \mathrm{V})}{\sigma_{1}^{2}(\mathrm{~V})+\sigma_{1}(\mathrm{X}, \mathrm{V})} \\
& \gamma_{2}=\frac{\sigma_{2}^{2}(\mathbf{Y})+\sigma_{2}(\mathrm{Y}, \mathrm{V})}{\sigma_{2}^{2}(\mathrm{~V})+\sigma_{2}(\mathbf{Y}, \mathbf{V})}
\end{aligned}
$$


Data from Forms $\mathrm{X}$ and $\mathrm{Y}$ of a 125 -item professional certification examination were used in this example. Form $X$ was the new form, and Form $Y$ was the old form. The two forms contained a common set of 30 items designated as $\mathrm{V}$. The scores of the common items contributed to the total scores. Form X was administered to a sample of 773 examinees, and Form $\mathrm{Y}$ was administered to 795 examinees. The two forms were administered one year apart. This dataset also was used in Kolen (1985). The descriptive statistics of the score distributions of the two test forms are presented in Table 3. The score distributions were negatively skewed.

Table 3

Score Summary Statistics for Forms $\mathrm{X}$ and $\mathrm{Y}$ and Common Items $\mathrm{V}$

\begin{tabular}{cccccc}
\hline \hline Score & Group & Mean & SD & Skewness & Kurtosis \\
\hline X & 1 & 95.75 & 13.38 & -1.03 & 3.91 \\
Y & 2 & 96.84 & 13.37 & -1.00 & 3.89 \\
V & 1 & 23.18 & 4.05 & -.84 & 3.48 \\
V & 2 & 22.54 & 4.31 & -.79 & 3.47 \\
\hline
\end{tabular}

Values of the first partial derivatives with respect to each of the 10 central moments involved in the Tucker equating function are shown in Table 4. These partial derivatives were evaluated at a score level of 80 using the analytical formulas derived by Kolen (1985). The bias of the numerical derivatives computed with the three-point and the five-point approximation formulas (Equations 2 and 4, respectively) also is presented in Table 4.

Table 4

First Partial Derivatives (at $x=80$ ) and Bias of Estimates for 3-Point and 5-Point Approximations

\begin{tabular}{|c|c|c|c|}
\hline \multirow[b]{2}{*}{ Moment } & \multirow{2}{*}{$\begin{array}{c}\text { Exact } \\
\text { Derivative }\end{array}$} & \multicolumn{2}{|c|}{ Bias of Estimate } \\
\hline & & 3-Point & 5-Point \\
\hline$\mu_{1}(\mathrm{X})$ & -.951 & $2.8 \times 10^{-17}$ & $2.8 \times 10^{-17}$ \\
\hline$\mu_{1}(V)$ & 2.764 & $-1.2 \times 10^{-7}$ & $-3.2 \times 10^{-12}$ \\
\hline$\sigma_{1}^{2}(\mathrm{X})$ & .037 & $2.1 \times 10^{-8}$ & $-5.9 \times 10^{-14}$ \\
\hline$\sigma_{1}^{2}(V)$ & -.414 & $-2.8 \times 10^{-7}$ & $1.2 \times 10^{-13}$ \\
\hline$\sigma_{1}(X, V)$ & .035 & $-1.6 \times 10^{-9}$ & $-2.3 \times 10^{-16}$ \\
\hline$\mu_{2}(\mathrm{Y})$ & 1.000 & $-2.0 \times 10^{-17}$ & $-1.4 \times 10^{-17}$ \\
\hline$\mu_{2}(V)$ & -2.764 & $1.2 \times 10^{-7}$ & $2.9 \times 10^{-12}$ \\
\hline$\sigma_{2}^{2}(Y)$ & -.041 & $-5.6 \times 10^{-9}$ & $1.1 \times 10^{-14}$ \\
\hline$\sigma_{2}^{2}(\mathrm{~V})$ & .233 & $3.9 \times 10^{-7}$ & $-2.3 \times 10^{-12}$ \\
\hline$\sigma_{2}(\mathrm{Y}, \mathrm{V})$ & .029 & $2.7 \times 10^{-10}$ & $2.6 \times 10^{-17}$ \\
\hline
\end{tabular}

The third- and higher-order partial derivatives of the Tucker equating function with respect to $\mu_{1}(\mathrm{X})$ and $\mu_{2}(\mathrm{Y})$ were 0 ; therefore, these two first partial derivatives derived numerically should be the exact values. Table 4 shows that the partial derivatives with respect to $\mu_{1}(X)$ and $\mu_{2}(Y)$ estimated with the three-point and five-point approximation formulas were essentially identical to the exact values (with the bias of estimate less than $10^{-16}$ ). For the partial derivatives with respect to the other moments, some errors were involved in the approximations, but these errors were very small. Table 4 shows that the maximum bias of the estimate was less than $10^{-6}$ for the three-point approximation and less than $10^{-11}$ for the five-point approximation. 
Table 5

Standard Errors of Tucker Equating and Bias of Estimates for 3-Point and 5-Point Approximations

\begin{tabular}{rcccc}
\hline \hline & & & \multicolumn{2}{c}{ Bias of Estimate } \\
\cline { 4 - 5 }$x$ & $\hat{l}(x)$ & SE[ $\hat{l}(x)]$ & 3-Point & \multicolumn{1}{c}{5 -Point } \\
\hline 40 & 45.577 & 1.419 & $2.2 \times 10^{-7}$ & $-1.9 \times 10^{-13}$ \\
50 & 55.092 & 1.190 & $1.7 \times 10^{-7}$ & $-1.4 \times 10^{-13}$ \\
60 & 64.606 & .965 & $1.3 \times 10^{-7}$ & $-8.7 \times 10^{-14}$ \\
70 & 74.121 & .744 & $8.9 \times 10^{-8}$ & $-3.9 \times 10^{-14}$ \\
80 & 83.635 & .536 & $4.7 \times 10^{-8}$ & $-6.9 \times 10^{-16}$ \\
90 & 93.150 & .361 & $1.0 \times 10^{-8}$ & $6.9 \times 10^{-15}$ \\
100 & 102.664 & .288 & $7.2 \times 10^{-9}$ & $-7.2 \times 10^{-14}$ \\
110 & 112.178 & .381 & $5.3 \times 10^{-8}$ & $-1.8 \times 10^{-13}$ \\
120 & 121.693 & .563 & $1.0 \times 10^{-7}$ & $-2.6 \times 10^{-13}$ \\
\hline
\end{tabular}

Table 5 shows the SEs of Tucker equating at selected score levels $(x)$ computed using the exact derivatives (see Table 4) and the bias of the estimates using the numerical derivatives. These results indicate that the numerical approach was very accurate. The maximum difference between the SEs computed using the three-point approximation derivatives and those using the exact derivatives was less than $10^{-6}$. The results from the five-point numerical approach and the analytical approach were indistinguishable (with the maximum bias of the estimate less than $10^{-12}$ ).

\section{Computer Simulation}

\section{Method}

The numerical approach was used to compute the SES of Levine observed score equating (also called Levine equally reliable equating) with an external set of common items. Due to the complicated form of the Levine observed score equating function with external common items (see Kolen \& Brennan, 1987), the first partial derivatives are very difficult to derive analytically. The advantage of the numerical approach is apparent in such a case. The Levine observed score equating function is given in Table 2.

The numerically derived SES of Levine equating were investigated in a computer simulation in which the numerically derived SEs were compared to the estimated true values. Suppose two test forms, Form $\mathrm{X}$ and Form $\mathrm{Y}$, are to be equated. Designate Form $\mathrm{X}$ as the new form and Form $\mathrm{Y}$ as the old form. Both forms contain a set of common items designated as $\mathrm{V}$, which is treated as external. That is, the score for $\mathrm{V}$ is not counted in the total test score.

To estimate the true SE of equating, a pair of population bivariate distributions of test scores must be specified so that a large number of random samples can be drawn from the specified populations. Here, two population bivariate distributions of test scores for Form X with V and Form $\mathrm{Y}$ with $\mathrm{V}$ were estimated by fitting the data used in the previous example with a bivariate log-linear model (Agresti, 1990, sections 13.1 and 13.2). A computer program written by Hanson (1991) was used. Because $\mathrm{V}$ was external, scores on $\mathrm{V}$ were not counted in the total test scores.

The log-linear model fit the data in such a way that the first five moments of each marginal distribution and the correlation between scores of equating items and nonequating items were the same for the fitted and observed distributions. The likelihood ratio $\chi^{2}$ was 887.26 [with 2,962 degrees of freedom $(d f)$ and $p>$.99] for fitting the bivariate distribution of the Form $\mathrm{X}$ data. The likelihood ratio $\chi^{2}$ was 918.45 (with 2,964 $d f$ and $p>$.99) for fitting the Form $Y$ data. These fit statistics suggested that the model fit the data quite well. The simulation was conducted using the following steps. 
Step 1. A pair of bivariate distributions were generated from the score distributions of the specified populations. This pair of distributions represented observed score distributions obtained from administering Form X (with V) and Form Y (with V) to a pair of random samples of size $n$ drawn from two populations of examinees under the CING design and determining the scores from the two samples on $\mathrm{V}$ (the set of $\mathbf{3 0}$ common items). Levine observed score equating was performed with the randomly generated score distributions. Form Y equivalents of Form X scores and SEs with and without the normality assumption were computed for all integer score points between 0 and 95 . The partial derivatives were computed using the three-point approximation formula.

Step 2. Step 1 was replicated 5,000 times. The means and standard deviations (SDs) of Form Y equivalents and SEs derived with and without the normality assumption were computed for all score points from the values over the 5,000 replications.

The true SE of equating for a score point $x$ was computed as

$\mathrm{SE}=\left(\frac{\sum_{i=1}^{5,000}\left[l(x)-\hat{l}_{i}(x)\right]^{2}}{5,000}\right)^{1 / 2}$,

where

$l(x)=\frac{\sum_{i=1}^{5,000} \hat{l}_{i}(x)}{5,000}$

was used to estimate $l(x)$.

The bias and variability of the numerical SEs derived with and without the normality assumption were investigated by comparing the estimated values to the true values. The simulation was conducted with different sample sizes $(n=100,200,400,1,000$, and 2,000). Average absolute bias, defined as

$\operatorname{BIAS}=\sum_{x=0}^{95}|\operatorname{SE}[\hat{l}(x)]-\operatorname{SEE}[\hat{l}(x)]| \mathbf{P}(x)$,

was computed across all score points for each sample size, where

$\mathrm{SE}[\hat{l}(x)]$ is the true SE at a score level of $x$,

$\hat{S E}[\hat{l}(x)]$ is the average estimate over the 5,000 replications, and

$\mathrm{P}(x)$ is the proportion of examinees in the population taking the new form who had a score of $x$.

\section{Results and Discussion}

The results of the simulation are presented in Table 6. The estimate of the Form Y equivalent for each selected score point of Form $\mathrm{X}$ is the average value of $\hat{l}(x)$ over the 5,000 replications. Because a very large number of replications was used, this estimate was quite stable. The different sample sizes had little effect on the estimated Form Y equivalents. For $n=1,000$ and $n=2,000$, the estimated Form $Y$ equivalents were almost identical.

The Form Y equivalent $\hat{l}(x)$ for a particular Form $\mathrm{X}$ score was computed from a pair of random samples of examinees drawn from the populations. Random errors due to sampling were, therefore, involved in $\hat{l}(x)$. Suppose an infinite number of random samples are drawn from the population and $\hat{l}(x)$ is computed for each sample; then the SD of the $\hat{l}(x)$ distribution is the SE of the Form Y equivalent of score $x$. In the simulation, a large finite number of random samples was used to estimate 
Table 6

Results of Computer Simulation, With and Without Normality Assumption, for $x=40,50,60,70$, and 80, and Average Absolute Bias (AAB), for $n=100$ to 2,000

\begin{tabular}{|c|c|c|c|c|c|c|}
\hline \multirow[b]{2}{*}{$n$ and $x$} & \multirow[b]{2}{*}{$\hat{l}(x)$} & \multirow[b]{2}{*}{$\operatorname{SE}[\hat{l}(x)]$} & \multicolumn{2}{|c|}{$\operatorname{SE}[\hat{l}(x)]-\hat{\operatorname{SE}}[\hat{l}(x)]$} & \multicolumn{2}{|c|}{ SD of Estimates } \\
\hline & & & Normal & Non-Normal & Normal & Non-Normal \\
\hline \multicolumn{7}{|l|}{$n=100$} \\
\hline 40 & 47.04 & 8.587 & -1.589 & -.649 & 2.678 & 3.083 \\
\hline 50 & 55.57 & 6.108 & -1.248 & -.446 & 1.807 & 2.135 \\
\hline 60 & 64.11 & 3.752 & -.799 & -.238 & .952 & 1.201 \\
\hline 70 & 72.64 & 2.013 & .041 & -.049 & .400 & .423 \\
\hline 80 & 81.17 & 2.688 & .513 & -.109 & .941 & .837 \\
\hline AAB & & & .0256 & .0202 & & \\
\hline \multicolumn{7}{|l|}{$n=200$} \\
\hline 40 & 47.51 & 5.601 & -.859 & -.162 & 1.141 & 1.356 \\
\hline 50 & 55.90 & 3.994 & -.697 & -.109 & .771 & .943 \\
\hline 60 & 64.29 & 2.475 & -.462 & -.057 & .408 & .534 \\
\hline 70 & 72.68 & 1.382 & .046 & -.023 & .180 & .190 \\
\hline 80 & 81.07 & 1.815 & .387 & -.053 & .403 & .354 \\
\hline AAB & & & .0246 & .0072 & & \\
\hline \multicolumn{7}{|l|}{$n=400$} \\
\hline 40 & 47.66 & 3.778 & -.466 & .034 & .532 & .633 \\
\hline 50 & 56.01 & 2.702 & -.400 & .021 & .360 & .441 \\
\hline 60 & 64.37 & 1.686 & -.279 & .009 & .191 & .251 \\
\hline 70 & 72.72 & .952 & .052 & .001 & .085 & .090 \\
\hline 80 & 81.07 & 1.220 & .327 & .014 & .188 & .163 \\
\hline AAB & & & .0244 & .0019 & & \\
\hline \multicolumn{7}{|l|}{$n=1,000$} \\
\hline 40 & 47.77 & 2.459 & -.390 & -.071 & .211 & .250 \\
\hline 50 & 56.08 & 1.756 & -.317 & -.049 & .143 & .174 \\
\hline 60 & 64.39 & 1.091 & -.210 & -.027 & .075 & .099 \\
\hline 70 & 72.70 & .608 & .023 & -.009 & .034 & .036 \\
\hline 80 & 81.01 & .788 & .181 & -.017 & .075 & .064 \\
\hline AAB & & & .0116 & .0026 & & \\
\hline \multicolumn{7}{|l|}{$n=2,000$} \\
\hline 40 & 47.89 & 1.677 & -.223 & .002 & .102 & .121 \\
\hline 50 & 56.17 & 1.198 & -.187 & .002 & .069 & .085 \\
\hline 60 & 64.44 & .746 & -.127 & .002 & .036 & .048 \\
\hline 70 & 72.72 & .423 & .023 & -.001 & .016 & .017 \\
\hline 80 & 81.00 & .547 & .136 & -.004 & .036 & .031 \\
\hline $\mathrm{AAB}$ & & & .0096 & .0003 & & \\
\hline
\end{tabular}

the true SE of $\hat{l}(x)$. It was found that 5,000 random samples were enough to make the estimates very close to the true SEs. In this study, the estimates obtained from the simulation were treated as true SEs of equating and were used as criteria for evaluating the SEs derived numerically.

The results of the simulation (see Table 6) indicate that for a particular score point $(x)$, the SE of equating decreased as the sample size increased. This trend is consistent with the notion that if the sample size is infinitely large, then the expected SE of equating would be 0 . For a fixed sample size, the SES were smaller for score points near the mean than for score points at the two ends of the score range.

The bias of the numerically derived SEs of $\hat{l}(x)$ for each selected score point and the average absolute bias across all score points for each sample size are reported in Table 6. The SDs of estimation also are reported in Table 6 . In general, the bias of the estimate derived without the normality 
assumption was smaller than the bias of the estimate derived with the normality assumption. This result indicates that if the score distributions are not normal, then the SEs of equating derived with the normality assumption will be inaccurate and, therefore, the SEs derived without the normality assumption should be used. Similar results have been reported by Braun \& Holland (1982) and Kolen (1985).

Table 6 shows that with small sample sizes ( $n=100$ and $n=200$ ), the SEs derived numerically have large deviations from the true values, especially at the two ends of the score range. As sample size increases, the differences between the true and the numerically derived SEs become smaller. With larger sample sizes $(n=400$ and larger), the SEs derived without the normality assumption are very close to the true values. The SDS of the estimates show the same trend. Larger SDS of estimation are associated with smaller sample sizes. This trend is reasonable, because the variances and covariances of the moments used in computing the SEs of equating are based on large sample theory and are computed from higher-order central moments that are very sensitive to sampling errors. In fact, all the general delta methods that are not based on the normality assumption require large sample sizes. Otherwise, the obtained SEs might not be accurate.

The Levine observed score equating method requires some statistical assumptions. One important assumption is that the correlations between true scores on $\mathrm{X}$ and $\mathrm{Y}, \mathrm{X}$ and $\mathrm{V}$, and $\mathrm{Y}$ and $\mathrm{V}$ are all 1.0 (Kolen \& Brennan, 1987). In the present study, the assumptions required by the Levine method were not examined when deriving the population distributions. The SEs of equating studied here only reflect the equating error that is due to sampling error, and equating error due to the failure to meet the assumptions required by the equating method is not reflected in the SEs. Whether or not the simulated observed score distributions meet the assumptions required by the equating method should not affect the conclusion that can be reached from the simulation results about the precision of the estimated SES.

\section{Advantages and Usefulness of the Numerical Approach}

The advantage of the numerical approach for computing SEs of linear equating is that it is simple to implement. It is also fast in terms of computation speed, because there are no iterations involved in approximations. Each partial derivative is computed with two straight function evaluations if the three-point approximation is used, or with four straight function evaluations if the five-point approximation is used. Based on the implementation of the numerical approach on a Macintosh IICX computer, only a few seconds of time are needed to compute the equating function and the SES of equating from samples of 2,000 examinees.

The numerical approach is especially useful for computing SEs of complicated equating methods for which the analytical derivatives are not available or are too complicated to derive. A possible application is to apply the numerical approach to deriving SEs of a chain of equatings. Braun \& Holland (1982) presented a formula for computing the SEs of a chain of equatings with the assumption that each equating experiment conducted in the chain is statistically independent of all others. In many testing programs, especially those using the CING design, the equating experiments conducted in a chain are not statistically independent. In such situations, the SEs must be derived from the partial derivatives of the equating function and the moments of the score distributions. The analytical expressions of partial derivatives involved in a chain equating function are more complicated than those involved in a single equating. Use of the numerical approach can avoid this difficulty. 


\section{References}

Agresti, A. (1990). Categorical data analysis. New York: Wiley.

Angoff, W. H. (1971). Scales, norms, and equivalent scores. In R. L. Thorndike (Ed.), Educational measurement (2nd ed.; pp. 508-600). Washington DC: American Council on Education.

Braun, H. I., \& Holland, P. W. (1982). Observed-score test equating: A mathematical analysis of some ETS equating procedures. In P. W. Holland \& D. B. Rubin (Eds.), Test equating (pp. 9-49). New York: Academic Press.

Burden, R. L., \& Faires, J. D. (1985). Numerical analysis (3rd ed.). Boston MA: PWS-KENT Publishing.

Hanson, B. A. (1991). Using the MLLogLin function. Unpublished manuscript, Iowa City IA: American College Testing.

Kendall, M., \& Stuart, A. (1977). The advanced theory of statistics (4th ed., Vol. 1). New York: Macmillan.

Kolen, M. J. (1985). Standard errors of Tucker equating. Applied Psychological Measurement, 9, 209-223.

Kolen, M. J., \& Brennan, R. L. (1987). Linear equating models for the common-item nonequivalentpopulations design. Applied Psychological Measure- ment, 11, 263-277.

Lord, F. M. (1950). Notes on comparable scales for test scores (Research Bulletin No. RB-50-48). Princeton NJ: Educational Testing Service.

Lord, F. M. (1975). Automated hypothesis tests and standard errors for nonstandard problems. The American Statistician, 29, 56-59.

\section{Acknowledgments}

Computer programs written by Bradley Hanson were used in this study to estimate the population distributions and to generate random samples for estimating the true standard errors. Michael Kolen provided technical help on this project. The author benefited from comments of two anonymous reviewers when revising the manuscript.

\section{Author's Address}

Send requests for reprints or further information to Lingiia Zeng, American College Testing, 2201 North Dodge Street, P.O. Box 168, Iowa City IA 52243, U.S.A. 